\title{
A New Trick to Make Short-Pulse Ion Beams
}

\author{
A new laser technique could lead to ultrashort-pulse, high-energy ion \\ beams for medical use.
}

By Mark Buchanan

1 ntense beams of atomic nuclei such as carbon can be used to precisely target tumor cells in cancer patients, especially those cells that are resistant to traditional radiotherapy. Using powerful laser pulses aimed at ultrathin targets, researchers have now demonstrated an improved method for creating carbon beams consisting of extremely short pulses, which are expected to be less damaging to healthy cells [1]. The experiments show that the carbon nuclei in the target material can be selectively accelerated with respect to the lighter hydrogen nuclei. The proof of concept suggests that with the expected advances in laser technology, the technique could

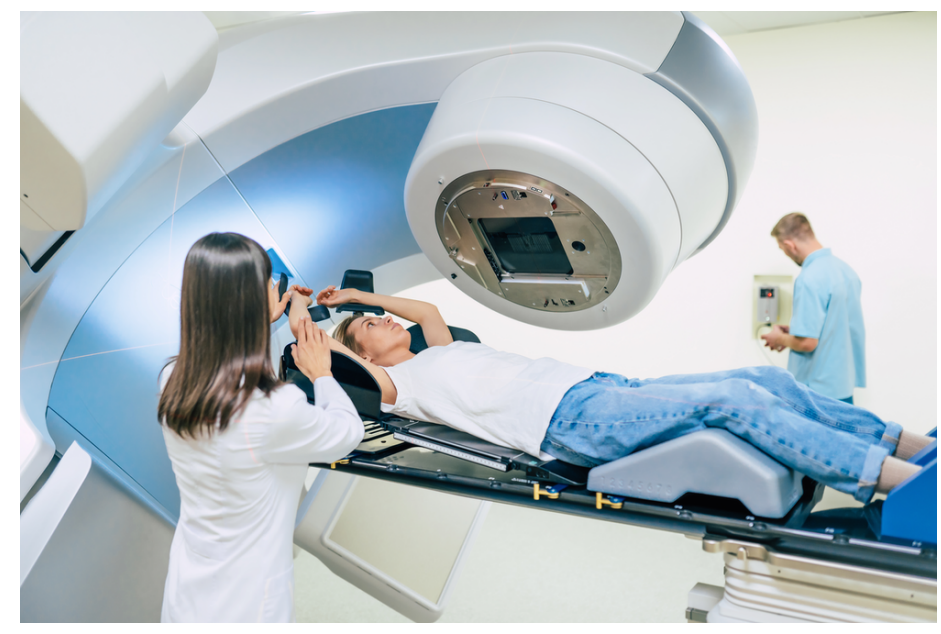

Life-saving beams. Standard treatments for cancer can involve a beam of $x$ rays or electrons from a linear accelerator like this one. New experiments demonstrate a technique that can produce ultrashort pulses of ions such as carbon, which may be less harmful to healthy cells than conventional beams.

Credit: M. Povozniuk/stock.adobe.com

lead to new and more versatile sources of high-energy ion beams for medical use.

In conventional cancer radiotherapy, $x$ rays or particle beams deliver the radiation dose to the tumor over several minutes. An emerging alternative approach aims to deliver doses over much shorter times, which may reduce damage to healthy cells. However, this approach has not yet been extended to ions larger than protons, even though some heavier ions, such as carbon nuclei, are very effective against certain tumor types.

One compact technique for rapidly accelerating ions involves hitting an ultrathin target with an intense laser pulse. The pulse vaporizes the material, creating a plasma of charged particles that the laser then accelerates on the far side of the target. However, in past attempts to use this so-called radiation pressure acceleration for ions other than protons, the plasma has typically contained both protons and heavier nuclei. The laser interaction generally accelerates the protons more easily, which diverts laser energy into protons. This effect has hampered previous efforts to create intense beams of carbon or other ions.

But Aodhan Mcllvenny of Queen's University Belfast in the UK and his colleagues have now found that a careful choice of the target thickness offers a way around this problem. Using the GEMINI laser at the Rutherford Appleton Laboratory in the UK, the researchers directed 40-femtosecond laser pulses onto targets of amorphous carbon, which contains hydrogen on its surface as a contaminant. Using targets ranging in thickness from 2 to 100 nanometers, the team focused the laser onto a spot roughly 3 micrometers in diameter. The plasma created in this material contains two species of ions-bare carbon nuclei 
and protons-which the team detected downstream.

Theoretical studies have predicted that there is an optimal target thickness for which radiation pressure produces the most effective acceleration, Mcllvenny says. In general, thinner targets should produce more energetic ions, as the laser has a smaller mass of ions to accelerate. However, if the target is too thin, the laser will pass through it without radiation pressure having a major effect. The experiments confirmed this prediction, as the carbon ions reached a maximum energy for 15-nanometer-thick targets. For these optimal targets, the researchers also found that the acceleration of protons dropped to a minimum. "This was a surprising result," says Mcllvenny, "and our previous models and assumptions couldn't account for it. Contrary to the norm, protons weren't dominating the acceleration process."

After running more experiments and performing computer simulations, the team found an explanation based on the shape of the laser pulses. The intensity of any real laser pulse grows gradually, rather than turning on instantaneously. Using computer simulations, Mcllvenny and his colleagues were able to show that-for their ideal target thickness-the early part of the pulse was strong enough to create the plasma and then to drive the protons out of it. This process left only carbon ions to be accelerated by the main portion of the pulse.

The researchers hope the technique can be refined further and can contribute to the development of more efficient cancer treatment. The team has already begun studying the effects of ultrafast carbon ion pulses on cancer cells that are resistant to conventional radiotherapy, says Mcllvenny, although the experiments are currently limited to two-dimensional arrays of cells. Available lasers produce carbon ion bursts with too little energy to penetrate most three-dimensional tissues, but as laser technology develops, the team expects to solve this problem.

"This is an exciting result," says plasma physicist Nigel Woolsey of York University, UK, who notes that hydrogen contamination has been a major problem afflicting previous laser techniques for accelerating ions. "Typically, everything we try to accelerate is contaminated with hydrocarbons. But this research demonstrates a way to remove the hydrogen from the target." Mark Buchanan is a freelance science writer who splits his time between Abergavenny, UK, and Notre Dame de Courson, France.

\section{REFERENCES}

1. A. Mcllvenny et al., "Selective ion acceleration by intense radiation pressure," Phys. Rev. Lett. 127, 194801 (2021). 